\title{
Dynamic Rocker-Bogie: Kinematical Analysis in a High-Speed Traversal Stability Enhancement
}

\author{
Sunxin Wang ${ }^{1,2}$ and Yan $\mathrm{Li}^{2}$ \\ ${ }^{1}$ School of Higher Vocational and Technical Education, Xian University of Technology, Xian 710082, China \\ ${ }^{2}$ School of Mechanical and Precision Instrument Engineering, Xi'an University of Technology, Xian 710048, China \\ Correspondence should be addressed to Sunxin Wang; wsx8280@126.com
}

Received 19 April 2016; Revised 2 August 2016; Accepted 23 August 2016

Academic Editor: Christopher J. Damaren

Copyright (c) 2016 S. Wang and Y. Li. This is an open access article distributed under the Creative Commons Attribution License, which permits unrestricted use, distribution, and reproduction in any medium, provided the original work is properly cited.

\begin{abstract}
The rocker-bogie suspension system has robust capabilities to deal with uneven terrain because of its distributing of the payload over its six wheels uniformly, while there is one major shortcoming to high-speed traversal over the planar terrain. This paper proposes a new dynamic rocker-bogie suspension system with two modes of operation: it can expand the span of the rocker-bogie support polygon to increase travel rate when the terrain is planar; and it can switch to its original configuration to move by low speed when it is faced with rough terrain. The analysis on dynamic stability margin and kinematical simulation on the two operating modes of rocker-bogie are employed to analyze and verify the rationality and effectiveness of the modification in the structure.
\end{abstract}

\section{Introduction}

All-terrain mobile robots are different from other ordinary mobile robots, because they consider the effect of unstructured terrain and its environment [1]. Therefore, these robots are designed to operate effectively on natural terrains that may be sloped, rough, and deformable [2] and are used in such fields as deep space exploration $[3,4]$, safety and rescue $[5,6]$, and military and civilian application $[7,8]$.

In order to ensure that the task is successfully achieved, it is a key issue to design and optimize the suspension system of all-terrain mobile robots to improve its mobile performance. Because of its robust capabilities to deal with obstacles, the rocker-bogie suspension system is successfully used in the Sojourner Mars Rover, MER (Mars Exploration Rover), and the latest MSL (Mars Science Laboratory) [9]. However, the rocker-bogie suspension system has still some shortcoming. First, the average speed of operation is very slow. Second, it makes the rocker-bogie system not suitable for situations where high-speed traversal over hard-flat surfaces is needed to cover large areas in short periods of time.

In pursuance of achieving a greater stability margin over high-speed traversal without losing the original configuration, a new dynamic rocker-bogie suspension system, with two modes of operation that can switch between original configuration and high-speed traversal configuration, is introduced in this paper. For high-speed traversal mode of rocker-bogie suspension system, it can expand the span of the rocker-bogie support polygon to increase speed when moving on hard-flat terrain or uneven terrain with minor obstacles. On the other hand, it can automatically switch to its original configuration to move by low speed when it is faced with rough terrain and significant obstacles.

The remaining parts of this paper are organized as follows. In Section 2, we propose modification in the structure of the rocker-bogie suspension system increasing the span of the support polygon. In Section 3, we analyze the stability margin of the structure modification by force-angle stability measure. The analysis and verification by the Adams simulation will be shown in Section 4. Finally, the conclusions are given in Section 5 .

\section{Modification in the Structure of the Rocker- Bogie Suspension System}

The rocker-bogie suspension system is a passive springless and symmetric mechanism. Each side of the rocker-bogie has a rocker and a bogie: the rocker is connected to the rear wheel, 


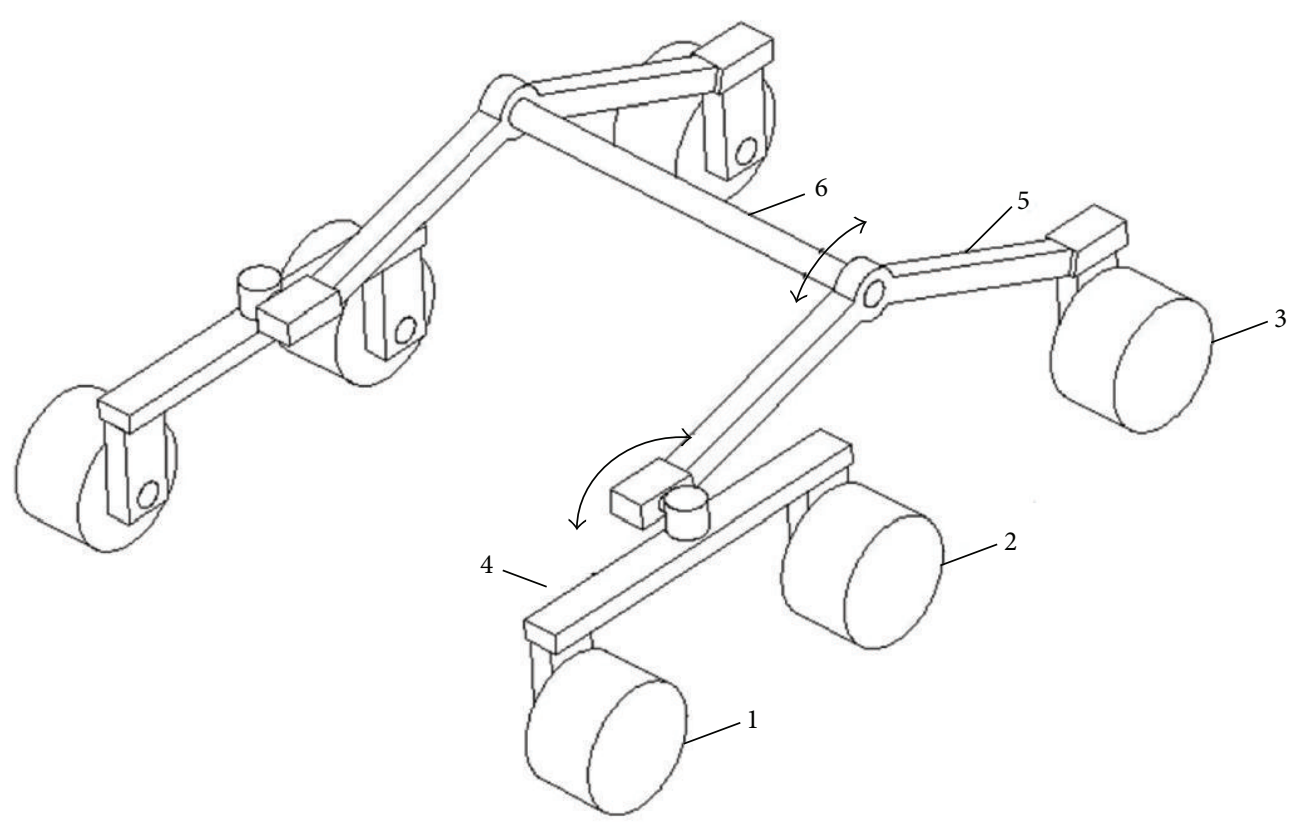

FIgURE 1: Rocker-bogie suspension system: 1, front wheel; 2, middle wheel; 3, rear wheel; 4, bogie; 5, rocker; 6, differential mechanism.

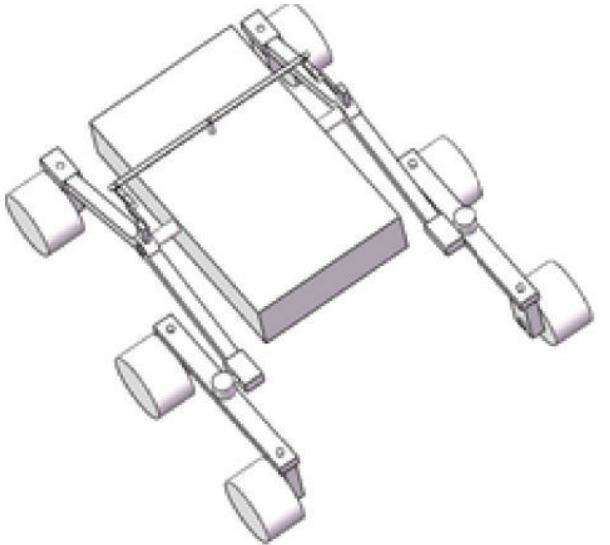

(a)

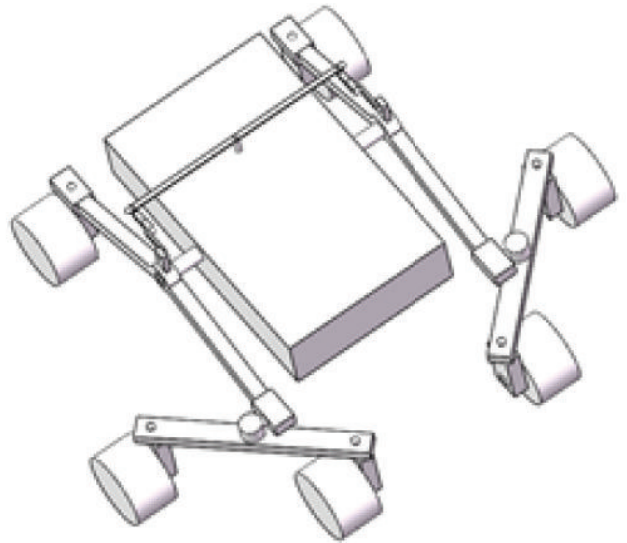

(b)

FIGURE 2: The two models of rocker-bogie suspension configurations: (a) original configuration and (b) high-speed traversal configuration.

and the middle wheel and the front wheel are connected by the bogie. The two sides of rocker-bogie are connected by the differential bar attached to the main body, which ensures that the six wheels are in contact with the ground all the time providing a stable platform for the scientific instruments and sensors, as shown in Figure 1.

The rocker-bogie suspension system is good at dealing with obstacles and excellent traversability. However, the rocker-bogie based robots must move at a very low average speed to ensure the stability of traveling [10]. In some situations, mobile robots mostly face slightly uneven terrain with rarely significant obstacles on it. This is why we proposed a configuration modification, expanding the span of the rocker-bogie system support polygon to increase the traversability. Nevertheless, when it needs to deal with obstacles, it can switch to its original configuration without losing its native robust capability. The three-dimensional model of the original configuration and the proposed highspeed traversal configuration is introduced in Figure 2.

As Figure 2 shows, the original configuration can only rotate in a pitch axis located at the front part of the rocker. The modification structure in the rocker-bogie suspension system is based on adding a rotation axis over the $Y$-plane of the bogie, varying the yaw orientation of the bogie. Therefore, the new high-speed traversal mechanism can alter the position of the outer support polygon points and increase the size of the area in contact with the ground.

This modification in configuration can achieve a flexible and efficient trafficability: one rocker-bogie system, two modes of operation. When the mobile robot faces significant 


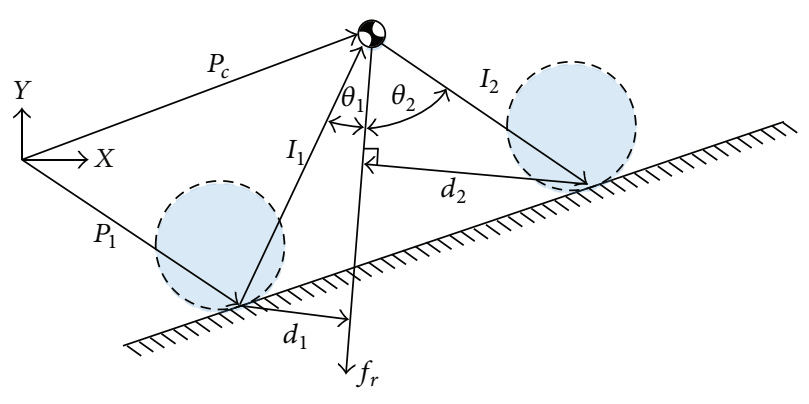

FIGURE 3: Planar force-angle stability metric.

obstacles, it needs to keep the original configuration and overcome them with a low speed to ensure the stability. When the mobile robot faces hard-flat terrain or slightly uneven terrain, it will switch to the high-speed traversal configuration by rotating the bogie over the $X$-plane, keeping excellent stability when moving at high speeds.

\section{Analysis on Dynamic Stability Margin for the High-Speed Traversal Enhancement}

As mentioned in Section 2, the new high-speed traversal mechanism is based on adding a rotation axis over the $Y$ plane of the bogie to expand its support polygon, making it more stable and adaptable while moving at high speed. And then, how many angles of rotating the bogie are optimal for the required high-speed mode position? As it was analyzed by using the Static Stability Factor (SSF) metric [11] and verified by the Solidworks software simulation in [12], once the system has rotated its bogies 45 degrees to the required high-speed mode position, the rover's support polygon is expanded reaching a bigger contact area.

The SSF metric employed in [12] is purely geometrical and suits for analyzing and evaluating the static stability margin, but it does not take any dynamic effects into consideration. In order to further analyze the effectiveness of our highspeed traversal configuration by dynamic stability margin, we select the force-angle stability metric [13] to replace the SSF metric. This new metric focuses its stability criterion on the tipover effect which occurs when a mobile system undergoes a reduction of ground contact points due to rotation.

A planar example that highlights the graphical nature of the force-angle stability metric is demonstrated by Figure 3. The Center of Mass (CM) of the planar system is subject to a net force $f_{r}$, which is the sum of all forces acting on the mobile system except the supporting reaction forces. This force vector subtends two angles, $\theta_{1}$ and $\theta_{2}$, with the two tipover axis normals $I_{1}$ and $I_{2}$ and acts along a line which is at a distance of $\left\|d_{1}\right\|$ and $\left\|d_{2}\right\|$, respectively, from the two tipover axes. The force-angle stability metric $\beta_{i}$ is given by the product of $\theta_{i},\left\|d_{i}\right\|$, and $\left\|f_{r}\right\|$, as formulated in the following:

$$
\beta_{i}=\theta_{i} \cdot\left\|d_{i}\right\| \cdot\left\|f_{r}\right\| .
$$

The system's stability margin $\beta_{S}$ is obtained by the minimum value of $\beta_{i}$. For a mobile system, if we can increase the value of the parameters involved in (1), the value of $\beta_{S}$ will

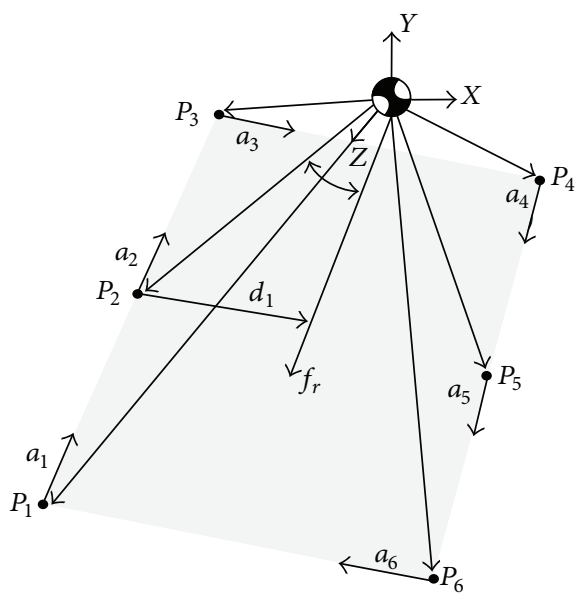

(a)

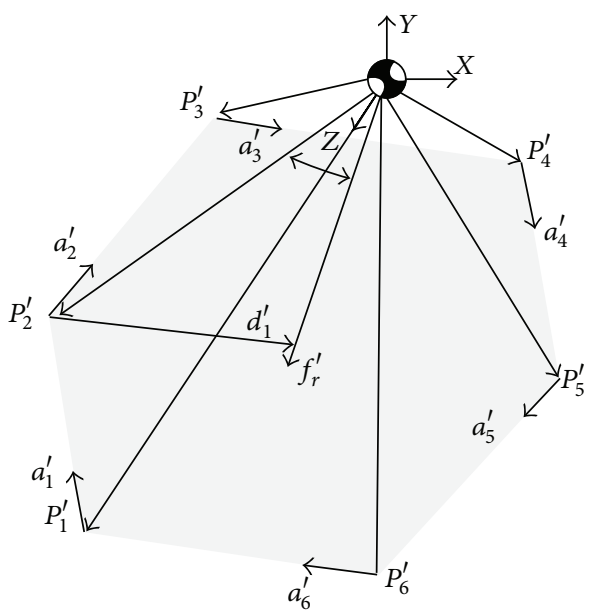

(b)

FIGURE 4: 3D force-angle stability metric: (a) original configuration and (b) high-speed traversal configuration.

get bigger, and the stability margin of the system will improve making the vehicle able to travel with higher stability.

The critical tipover stability occurs when $\beta_{S}$ goes to zero, that is, when any $\theta_{i},\left\|d_{i}\right\|$, or $f_{r}$ becomes zero. The angle $\theta_{i}$ becomes zero when the net force is coplanar with one of the tipover axes $I_{i}$, and this is the typical manner in which a tipover instability occurs. If $f_{r}$ lies outside the cone described by $I_{1}$ and $I_{2}$, the angle becomes negative and tipover is in progress. The distance $\left\|d_{i}\right\|$ becomes zero as the net force is coplanar with one of the tipover axes or as the system $\mathrm{CM}$ approaches one of the tipover axes. Note that these two geometric parameters, $\theta_{i}$ and $\left\|d_{i}\right\|$, together characterize the tipover stability margin of the system [14].

Of all the vehicle contact points with the ground, it is only necessary to consider those outermost points which form a convex support polygon when projected onto a horizontal plane, which is wheel-terrain contact point [14]. Therefore, we built the $3 \mathrm{D}$ force-angle stability measure diagram of the original configuration and the high-speed traversal configuration, respectively, as shown in Figure 4. 
For a consistent formulation, the $p_{i}$ parameters are numbered in ascending order following a right-hand rule where the thumb is directed downwards along the gravity vector; that is, the ground contact points are numbered in clockwise order when viewed from above. The lines which join the ground contact points are the candidate tipover mode axes, $a_{i}$, and the set of these lines will be referred to as the support polygon.

In order to increase the system's stability margin $\beta_{S}$, we should maximize the value of the product of $\theta_{i},\left\|d_{i}\right\|$, and $\left\|f_{r}\right\|$. As shown in Figure 4, the force-angle stability metric of the middle wheel is the minimum value, as we proposed previously. Therefore, we select the middle wheel of the two configurations as a focus point.

In the same condition, the value of the resultant force that acts on the original configuration, $\left\|f_{r}\right\|$, is equal to the value of the resultant force that acts on the high-speed traversal configuration $\left\|f_{r}^{\prime}\right\|$. However, the included angle $\theta_{1}$ and the vertical dimension of original configuration $\left\|d_{1}\right\|$ are smaller than the values $\theta_{1}^{\prime}$ and $\left\|d_{1}^{\prime}\right\|$ of the high-speed traversal configuration; therefore, the stability margin of the original configuration, $\beta_{S}$, is smaller than the high-speed traversal configuration, $\beta_{S}^{\prime}$.

Based on the analysis above, the rocker-bogie suspension mechanism has a greater stability margin after the high-speed traversal enhancement, making it more stable while traveling at high speed. Moreover, when the 45-degree rotation of the bogie is completed, the rover's support polygon is expanded reaching a bigger contact area [12]. This expansion of the contact area sets the rover's Center of Mass (CM) inside a bigger track base, making it more robust against load transfers due to the interaction of internal and external forces.

\section{Kinematical Simulation on the Two Operating Modes of Rocker-Bogie}

In paper [12], the two operating modes of rocker-bogie were tested by the Solidworks software, using their multibody dynamic motion analysis; thus, the data obtained of these simulations was contrasted with the static stability margin (i.e., Center of Gravity position) results.

In order to implement the kinematical simulation on rocker-bogie system, we use the Adams software to analysis and verify the two operating modes of rocker-bogie in two predefined scenarios: Scenario 1, where the terrain presents obstacles of heights smaller than the vehicle wheel's radius; Scenario 2, where the terrain is mostly covered in obstacles that surpass the previously mentioned vehicle wheel's radius.

Moreover, in order to comprehensively measure and verify the tipover stability of the two operating modes of rocker-bogie on uneven terrain, the rocker-bogie system's stability is expressed and evaluated in relation to each axis: lateral stability (stability in roll), directional stability (stability in yaw), and longitudinal stability (stability in pitch).

4.1. Simulation Results of Scenario 1. The 3D model was developed by Adams, as shown in Figure 5. We set up four segmented obstacles distributed on a flat surface, the height

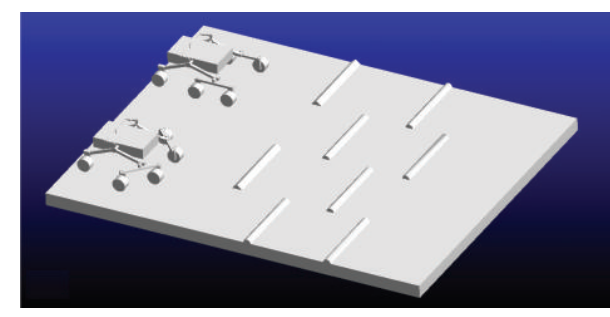

FIGURE 5: Kinematical simulation in scenario 1.

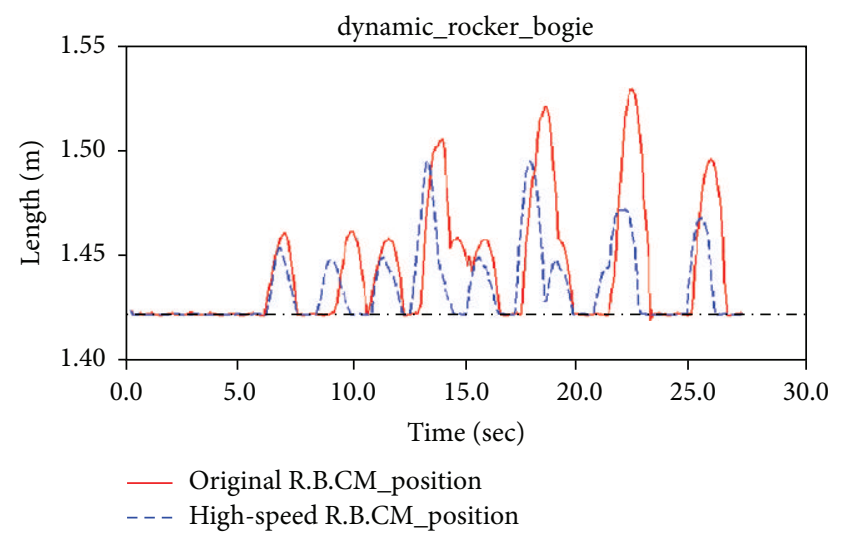

Figure 6: Vertical position variation of the vehicle's CM.

TABLE 1: Simulation parameters of the rocker-bogie suspension.

\begin{tabular}{lc}
\hline Parameters & Value \\
\hline Integral dimension $(\mathrm{mm})$ & $2760 * 2300 * 1015$ \\
Total weight $(\mathrm{Kg})$ & 330 (no-load) \\
Wheel diameter $(\mathrm{mm})$ & 500 \\
Wheel width $(\mathrm{mm})$ & 350 \\
Damping & 1.5 \\
Stiffness coefficient $(\mathrm{N} / \mathrm{mm})$ & $10^{5}$ \\
Dynamic friction coefficient: $f_{d}$ & 0.5 \\
Static friction coefficient: $f_{s}$ & 0.8 \\
Dynamic friction translation velocity: $V_{d}$ & 1.0 \\
(mm/s) & 0.1 \\
Static friction slip velocity: $V_{s}(\mathrm{~mm} / \mathrm{s})$ &
\end{tabular}

of every obstacle is $150 \mathrm{~mm}$, which is less than the radius of the wheel, and the wheel rotation speed is $100 \mathrm{deg} / \mathrm{s}$. The technical parameters of the rocker-bogie suspension are shown in Table 1. The dynamic performance curve is shown in Figures 7,8 , and 9. The solid red line in the graph describes the dynamic performance of original configuration; the dashed blue lines describe the dynamic performance of high-speed traversal configuration.

The vertical direction of the main body's Center of Mass is shown in Figure 6, and the data in Figure 6 is shown in Table 2. After 12 seconds of simulation run, all six wheels of the vehicle are engaging obstacles in the rugged topography of the terrain. 


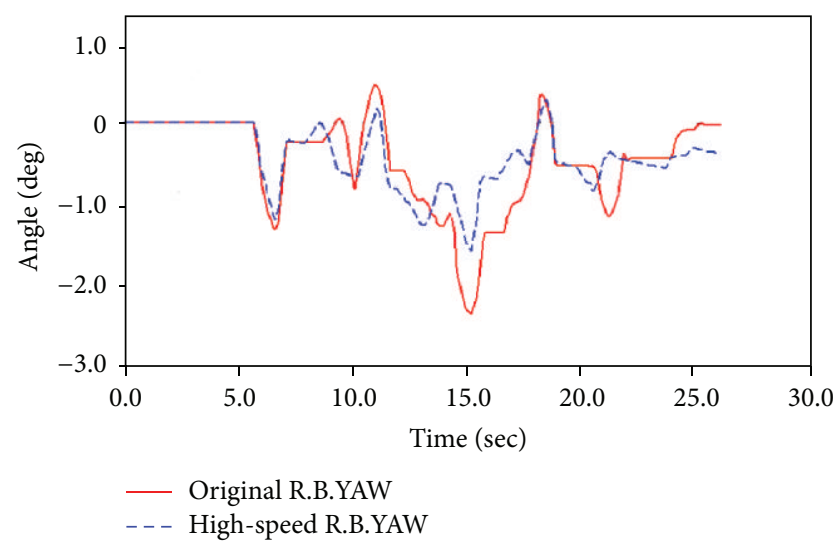

(a)

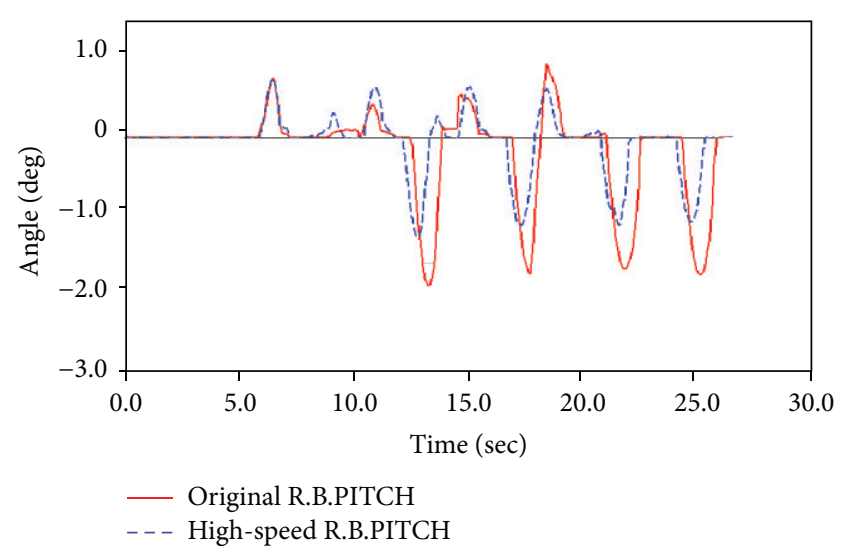

(b)

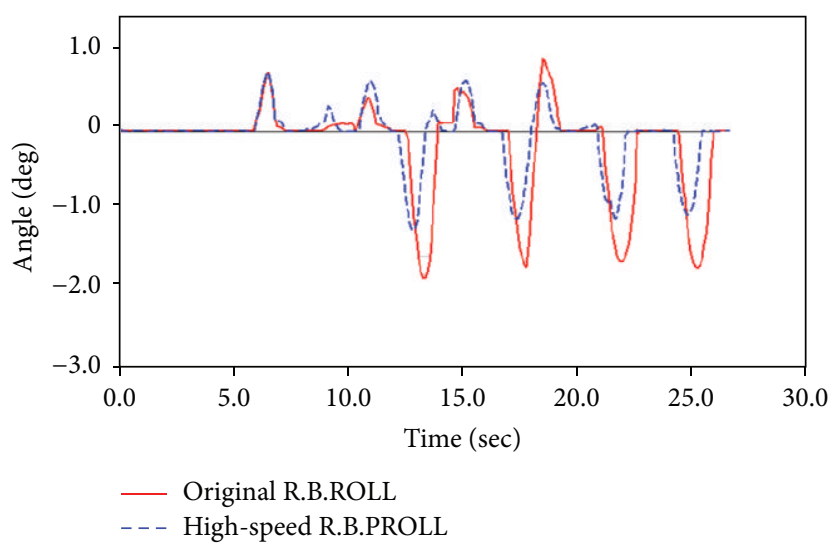

(c)

Figure 7: Yaw, pitch, and roll angular variation of the main body: (a) yaw angular variation of the main body, (b) pitch angular variation of the main body, and (c) roll angular variation of the main body.

TABLE 2: Data from the CM's vertical position variation.

\begin{tabular}{lcc}
\hline & Original conf. & $\begin{array}{c}\text { High-speed } \\
\text { traversal conf. }\end{array}$ \\
\hline $\begin{array}{l}\text { Minimum value of CM's } \\
\text { height (mm) }\end{array}$ & 1420.65 & 1420.64 \\
$\begin{array}{l}\text { Maximum value of CM's } \\
\text { height (mm) } \\
\text { CM's height variation range } \\
(\mathrm{mm})\end{array}$ & 1525.57 & 1490.98 \\
\hline
\end{tabular}

The variation of CM displacement in the process is the basis of the stability evaluation of all-terrain mobile robots, which will generally affect the stability of the structure. From the table, the alterations of CM displacement for original configuration are one point five times those of high-speed traversal configuration. Overall, the latter perform better.

The curve of the yaw, pitch, and roll angular variation of the main body is shown in Figure 7 and the data in Figure 7 is shown in Table 3.

The variation ranges of yaw, pitch, and roll in the process directly affect the vehicle stability, as the result shows that the three-variation range is about $50 \%$ higher than that of high-speed mode. The smaller the angle change, the little the probability that the vehicle will tip over.

The variation of velocity, acceleration, angular velocity, and angular acceleration of the main body is shown in Figure 8, and the data in Figure 8 is shown in Table 4 .

Through contrast analysis, we can see that the all indicators variation ranges of the rocker-bogie high-speed traversal configuration are smaller than the one of the rocker-bogie original configuration, and the curve of the high-speed configuration changes more gently without violent alterations, while in the curve of the original configuration there exist significant alterations, which may cause the vehicle to tip over. Thus, we draw the conclusion that the high-speed traversal configuration is more stable than the original configuration, making it more suitable for this proposed scenario.

4.2. Simulation Results of Scenario 2. Traversability reflects the mobile robot's ability to adapt to significant rough terrains. Mobile robots will be challenged with various obstacles on the road, with the ones that are perpendicular to the surface being the most severe. Therefore, the ability of the mobile robot to overcome vertical obstacles reflects its traversability capabilities. For this purpose, we developed the 


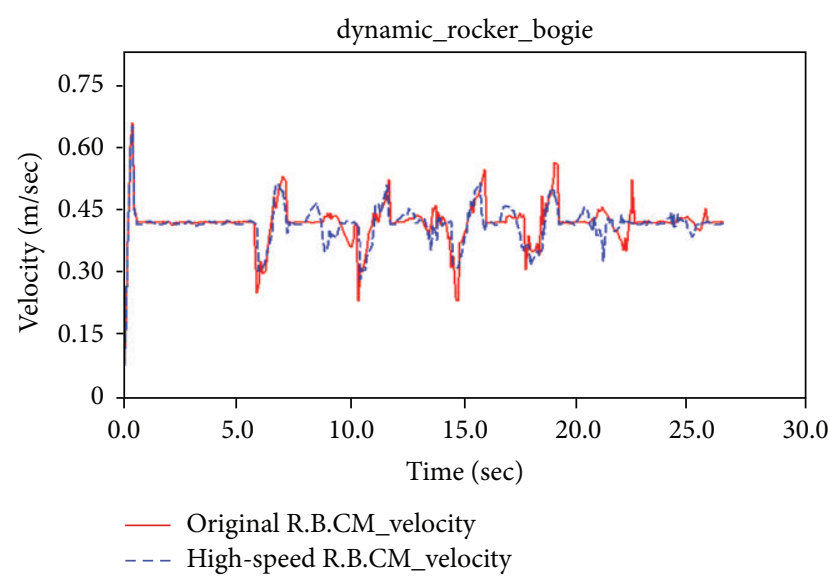

(a)

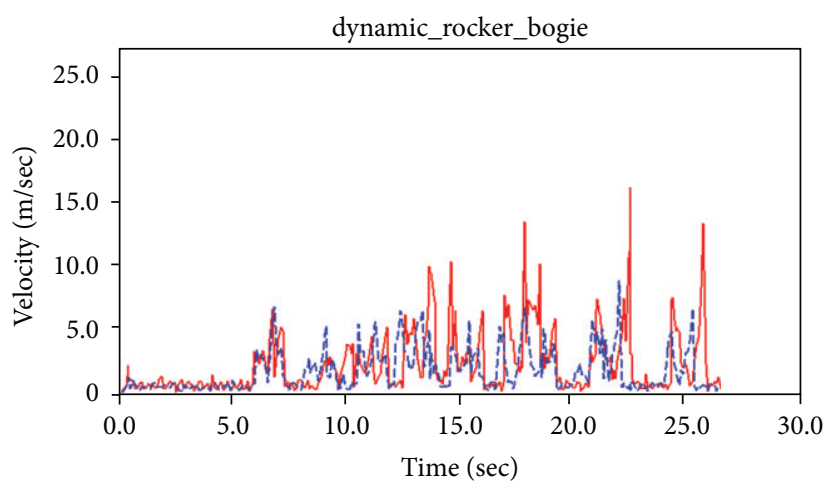

- Original R.B.CM_angular_velocity
- - High-speed R.B.CM_angular_velocity

(c)

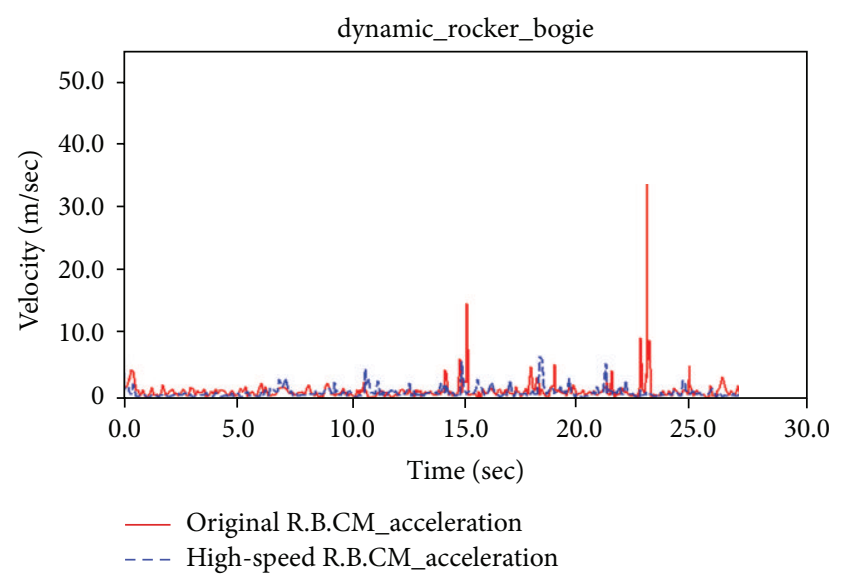

(b)

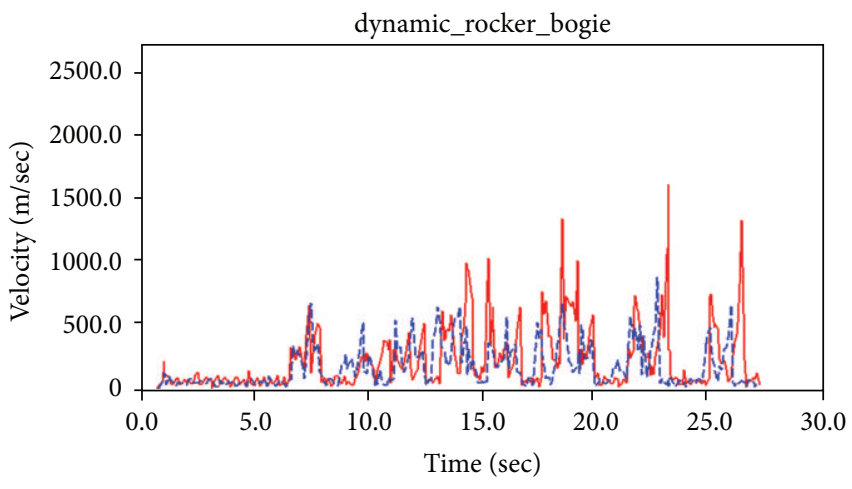

— Original R.B.CM_angular_acceleration

- - - High-speed R.B.CM_angular_acceleration

(d)

FIGURE 8: Velocity, acceleration, angular velocity, and angular acceleration variation of the main body: (a) velocity variation of the main body, (b) acceleration variation of the main body, (c) angular velocity variation of the main body, and (d) angular acceleration variation of the main body.

TABLE 3: Data from yaw, pitch, and roll angular variation.

\begin{tabular}{lcc}
\hline & Original conf. & High-speed traversal conf. \\
\hline Minimum value of yaw angle $\left(^{\circ}\right)$ & -2.65 & -1.77 \\
Maximum value of yaw angle $\left(^{\circ}\right)$ & 0.52 & 0.21 \\
Yaw angle variation range $\left(^{\circ}\right)$ & 3.17 & 1.98 \\
Minimum value of pitch angle $\left(^{\circ}\right)$ & -4.11 & -2.75 \\
Maximum value of pitch angle $\left(^{\circ}\right)$ & 2.03 & 1.65 \\
Pitch angle variation range $\left(^{\circ}\right)$ & 6.14 & 4.40 \\
Minimum value of roll angle $\left(^{\circ}\right)$ & -1.76 & -1.19 \\
Maximum value of roll angle $\left(^{\circ}\right)$ & 1.63 & 1.07 \\
Roll angle variation range $\left({ }^{\circ}\right)$ & 3.39 & 2.26 \\
\hline
\end{tabular}

terrain shown in Figure 9. Every obstacle is perpendicular to the ground; the first barrier's height is $180 \mathrm{~mm}$, and then every barrier's height is $20 \mathrm{~mm}$ taller than the previous one. The technical parameters of the rocker-bogie suspension are the same as the ones previously mentioned in Section 4.1.

The final state of the simulation is shown in Figure 10. The high-speed traversal configuration can cross the barrier of
$280 \mathrm{~mm}$, but the bogie overturned when crossing the barrier of $300 \mathrm{~mm}$; as a result, the robot can no longer go any further. However, the original configuration can cross the barrier of $400 \mathrm{~mm}$ without any tipover situation, but the rear wheels were stuck when climbing the barrier of $420 \mathrm{~mm}$.

The distance between the front wheel and the center of the bogie in the original configuration is longer compared 
TABLE 4: Data from the velocity, acceleration, angular velocity, and angular acceleration variation.

\begin{tabular}{lcc}
\hline & Original conf. & High-speed traversal conf. \\
\hline Minimum value of velocity $(\mathrm{m} / \mathrm{s})$ & 0.16 & 0.29 \\
Maximum value of velocity $(\mathrm{m} / \mathrm{s})$ & 0.62 & 0.54 \\
Velocity variation range $(\mathrm{m} / \mathrm{s})$ & 0.46 & 0.25 \\
Minimum value of acceleration $\left(\mathrm{m} / \mathrm{s}^{2}\right)$ & 0.06 & 0.33 \\
Maximum value of acceleration $\left(\mathrm{m} / \mathrm{s}^{2}\right)$ & 51.61 & 10.11 \\
Acceleration variation range $\left(\mathrm{m} / \mathrm{s}^{2}\right)$ & 51.55 & 9.78 \\
Minimum value of angular velocity $(\mathrm{deg} / \mathrm{s})$ & 0.02 & 0.07 \\
Maximum value of angular velocity $(\mathrm{deg} / \mathrm{s})$ & 20.65 & 11.25 \\
Angular velocity variation range $(\mathrm{deg} / \mathrm{s})$ & 20.63 & 11.18 \\
Minimum value of angular acceleration $\left(\mathrm{deg} / \mathrm{s}^{2}\right)$ & 7.54 & 21.55 \\
Maximum value of angular acceleration $\left(\mathrm{deg} / \mathrm{s}^{2}\right)$ & 1549.71 & 892.02 \\
Angular acceleration variation range $\left(\mathrm{deg} / \mathrm{s}^{2}\right)$ & 1542.17 & 870.47 \\
\hline
\end{tabular}

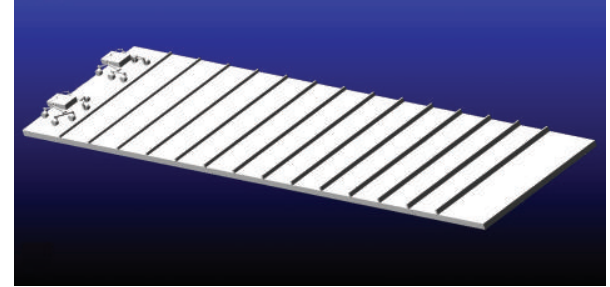

FIgURE 9: Kinematical simulation in scenario 2.

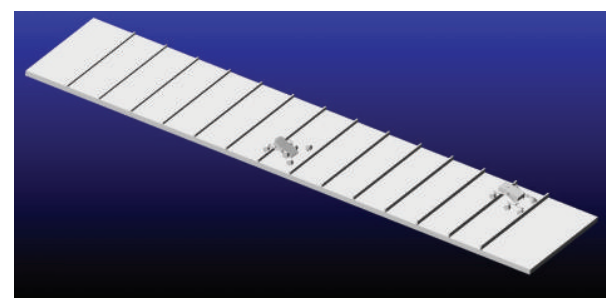

FIGURE 10: Final state of simulation in scenario 2.

to the one of the high-speed traversal configuration. The front wheel needs to have a greater force, which leads to significant variations of the front wheel's torque, causing vehicle overturn. We show in Figure 11 the variation of torque in the front wheel.

As we can see in Figure 11, when the obstacle height is lower than the wheel radius, the front wheel torques of the two configurations have differences but they not very significant. When the obstacle's height is gradually higher than the wheel's radius, the torque differences between the two configurations become more and more noticeable. The variation in the high-speed traversal configuration torque becomes more and more significant and violent; when the torque variation of the front wheel exceeds a certain limit, vehicle tipover happens.

Through the analysis from above, it is clear that the high-speed traversal configuration has better dynamic performance in terrains with minor obstacles, but when it faces

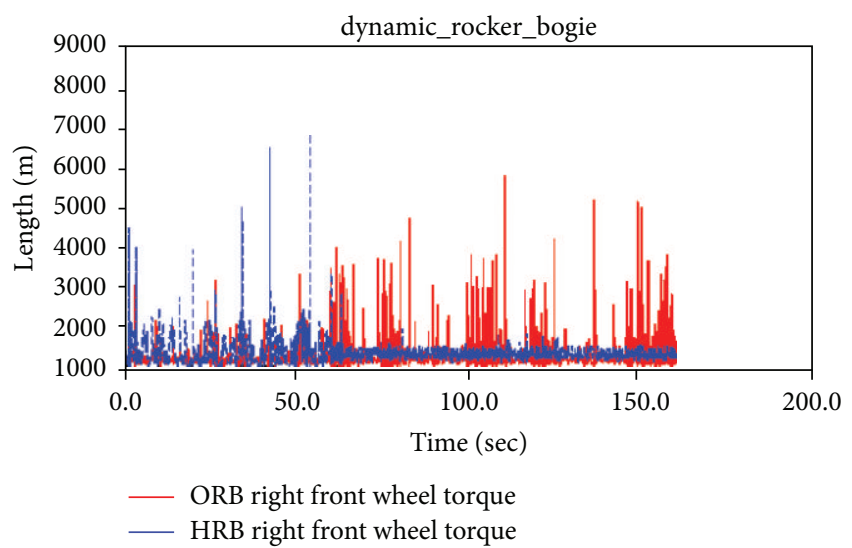

(a)

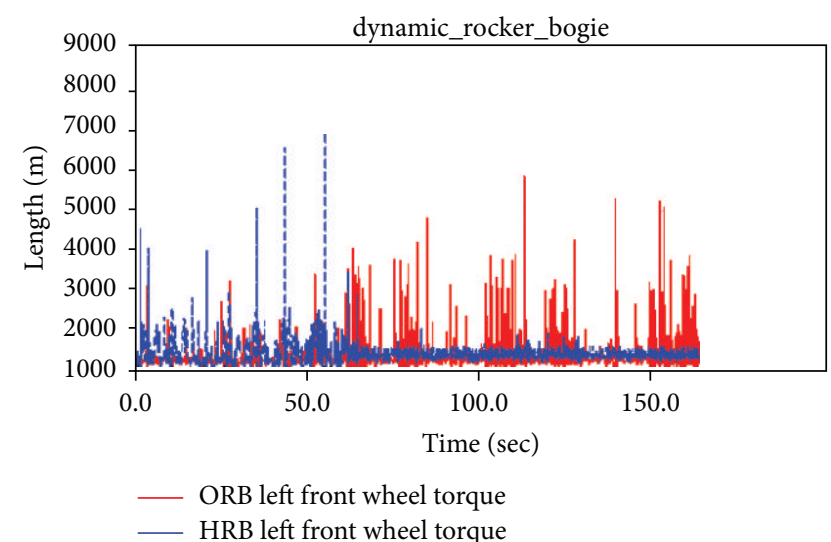

(b)

FIGURE 11: Torque variation of the front wheel: (a) torque variation of the right front wheel and (b) torque variation of the left front wheel.

significant obstacles, especially when the obstacle's height is bigger than wheel's radius, the original configuration has better performance than the high-speed traversal configuration, that is why we need to keep the two modes of operation. 


\section{Conclusion}

In this paper, our works focus on the two fields: (1) analysis of the dynamic stability margin for high-speed traversal enhancement of rocker-bogie; (2) kinematical simulation on the two operating mode of rocker-bogie. The idea, one rocker-bogie system with two modes of operation, is useful to increase the effectiveness and efficiency of the all-terrain mobile robots. This structural enhancement can make the rocker-bogie more adaptable and efficient in situations that require high-speed traversal or dealing with surfaces that need a more robust performance over tough obstacles.

As for the future work, we plan to extend our research to a field experimentations stage, using a testbed vehicle prototype to contrast and compare the obtained simulation results with practical observations of the proposed system's behavior, aiming to continue strengthening the vehicle's stability.

\section{Competing Interests}

The authors declare that they have no competing interests.

\section{References}

[1] S. C. Peters and K. Iagnemma, "Stability measurement of highspeed vehicles," Vehicle System Dynamics, vol. 47, no. 6, pp. 701$720,2009$.

[2] F. Michaud, D. Létourneau, M. Arsenault et al., "Multi-modal locomotion robotic platform using leg-track-wheel articulations," Autonomous Robots, vol. 18, no. 2, pp. 137-156, 2005.

[3] J. K. Erickson, "Living the dream-an overview of the Mars exploration project," IEEE Robotics \& Automation Magazine, vol. 13, no. 2, pp. 12-18, 2006.

[4] B. Chen, R. Wang, Y. Jia, L. Guo, and L. Yang, "Design of a high performance suspension for lunar rover based on evolution," Acta Astronautica, vol. 64, no. 9-10, pp. 925-934, 2009.

[5] A. Meghdari, H. N. Pishkenari, A. L. Gaskarimahalle, S. H. Mahboobi, and R. Karimi, "A novel approach for optimal design of a rover mechanism," Journal of Intelligent and Robotic Systems, vol. 44, no. 4, pp. 291-312, 2005.

[6] K. Nagatani, A. Yamasaki, Y. Kazuya, and T. Asachi, "Development and control method of six-wheel robot with rocker structure," in Proceedings of the IEEE International Workshop on IEEE Safety, Security and Rescue Robotics (SSRR '07), Rome, Italy, September 2007.

[7] F. Le Menn, P. Bidaud, and F. Ben Amar, "Generic differential kinematic modeling of articulated multi-monocycle mobile robots," in Proceedings of the IEEE International Conference on Robotics and Automation (ICRA '06), pp. 1505-1510, May 2006.

[8] C. Distante, G. Indiveri, and G. Reina, "An application of mobile robotics for olfactory monitoring of hazardous industrial sites," Industrial Robot, vol. 36, no. 1, pp. 51-59, 2009.

[9] T. Brooks, G. Gold, and N. Sertic, Dark Rover Rocker-Bogie Optimization Design, Thomas, 2011.

[10] D. P. Miller and T.-L. Lee, "High-speed traversal of rough terrain using a rocker-bogie mobility system," in Space, pp. 428-434, 2002.

[11] E. Garcia, J. Estremera, and P. Gonzalez de Santos, "A comparative study of stability margins for walking machines," Robotica, vol. 20, no. 6, pp. 595-606, 2002.
[12] H. A. Yang, L. C. V. Rojas, C. K. Xia, and Q. Guo, "Dynamic rocker-bogie: a stability enhancement for high-speed traversal," IAES International Journal of Robotics and Automation (IJRA), vol. 3, no. 3, pp. 212-220, 2014.

[13] E. G. Papadopoulos and D. A. Rey, "A new measure of tipover stability margin for mobile manipulators," in Proceedings of the 13th IEEE International Conference on Robotics and Automation, pp. 3111-3116, April 1996.

[14] E. Papadopoulos and D. A. Rey, "The force-angle measure of tipover stability margin for mobile manipulators," Vehicle System Dynamics, vol. 33, no. 1, pp. 29-48, 2000. 


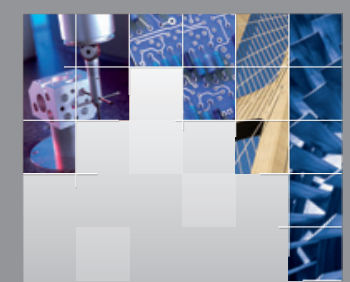

\section{Enfincering}
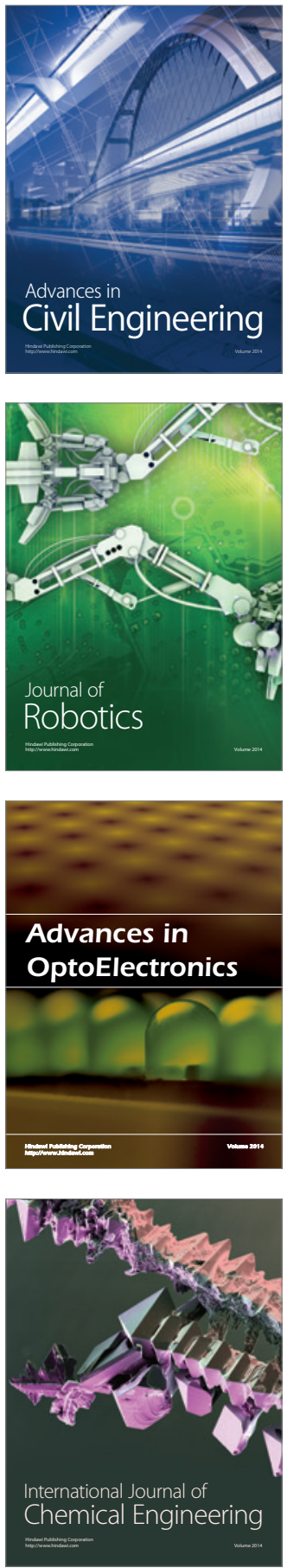

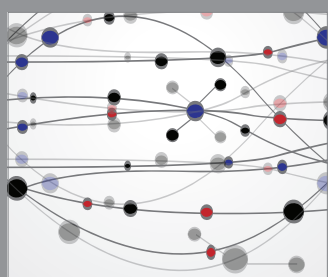

The Scientific World Journal

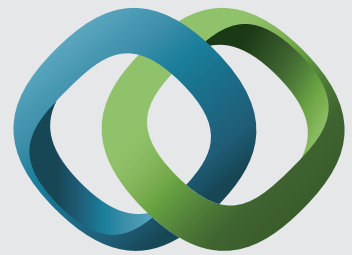

\section{Hindawi}

Submit your manuscripts at

http://www.hindawi.com
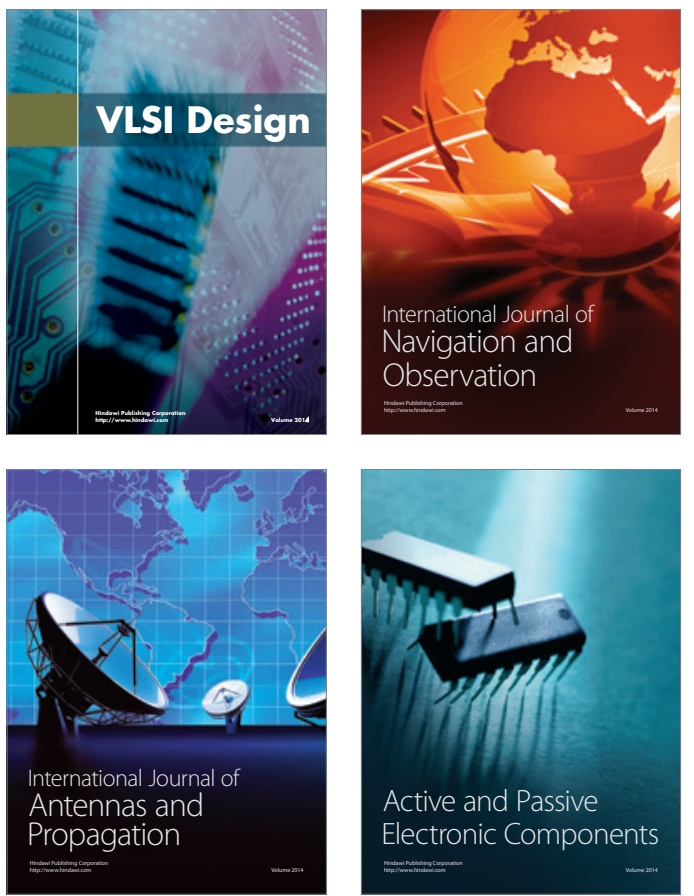
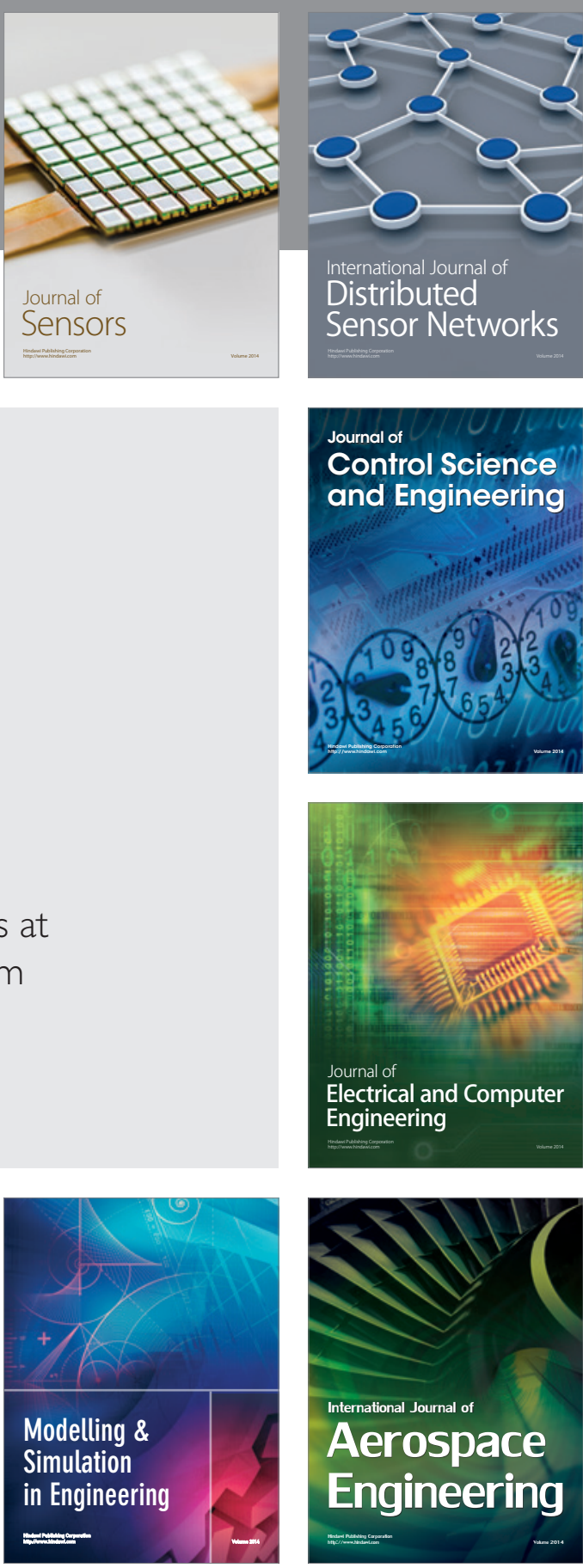

International Journal of

Distributed

Sensor Networks

Journal of

Control Science

and Engineering
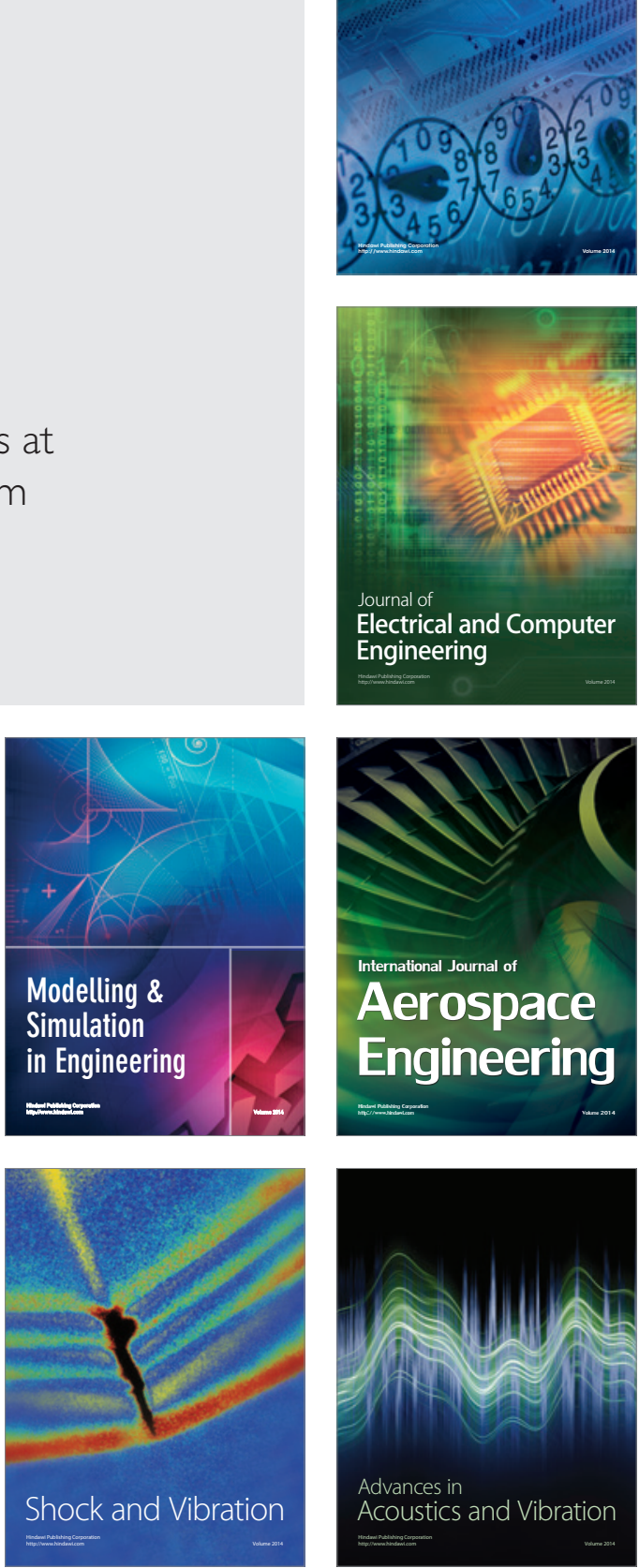